\title{
MICROCANTILEVER ARRAY SENSORS USING NANOPOROUS METAL-ORGANIC FRAMEWORKS (MOFS) FOR GAS DETECTION
}

\author{
Jin-Hwan Lee ${ }^{1}$, Ronald J. T. Houk ${ }^{2}$, Jeff A. Greathouse ${ }^{2}$, Mark D. Allendorf ${ }^{2}$, and Peter. J. Hesketh ${ }^{1}$ \\ ${ }^{1}$ G. W. Woodruff School of Mechanical Engineering, Georgia Institute of Technology, Atlanta, GA,USA \\ ${ }^{2}$ Sandia National Laboratories, Livermore, CA, USA
}

\begin{abstract}
This paper describes stress induced sensing mechanism with two different MOFs, specifically HKUST-1 and Cu(NDC)DABCO, on n-type piezoresistive microcantilever array. Thermomechanical responses of microcantilever sensors were characterized using water and methanol vapor in a designed gas test cell. The sensors exhibited substantially high sensitivity, 25.1 ohms/Mole \% for $\mathrm{H}_{2} \mathrm{O}$ with HKUST-1, at room temperature and 5 times faster response time with $\mathrm{Cu}(\mathrm{NDC}) \mathrm{DABCO}$ at elevated temperature. The key advantages of this new sensor are: 1 ) higher strain sensitivity due to n-type piezoresistors; 2) high selectivity with a nanoporous MOFs; 3) reversible response; and 4) possibility of multi-analyte detection due to array structure.
\end{abstract}

\section{INTRODUCTION}

There is a critical need for highly sensitive and selective low power chemical sensors for environmental monitoring, food safety, health diagnostics, security and defense. Detection is particularly critical when it is desured to know chemical weapons of mass destruction (CWMD), explosives, toxic industrial chemicals (TICs), and volatile organic compounds (VOCs). Although many sensitive transducer platforms have been developed, but the long-standing fundamental problem of sufficient sensitivity (sub-ppm) and selectivity of analytes has not yet been solved. The key component for accomplishing ultra-high sensitive sensors is an innovative improvement of sensor technology.

Metal-Organic Frameworks (MOFs) are flexible nanoporous materials with ultra high surface area and are attracting a great deal of attention for gas storage, gas purification and separation applications [1-9]. Metal organic frameworks (MOFs) are a diverse class of crystalline nanoporous materials with the potential to serve as highly tailorable recognition chemistries. Selective binding of $\mathrm{CO}_{2}$ vs. Ar, $\mathrm{N}_{2}$, or $\mathrm{CH}_{4}$ and $\mathrm{O}_{2}$ vs. $\mathrm{CO}$ by MOFs has been observed [1-3, 8-10]. There are many other MOFs involving a range of metals, linker groups, and pore sizes whose environments can be tailored to achieve specific molecular sorption properties. The permanent nanoporosity in MOFs results in exceptionally high surface areas in some cases $\left(>6,000 \mathrm{~m}^{2} / \mathrm{g}\right.$, the highest for any crystalline material) and thus the potential adsorb large quantities of gases. In fact, in addition to materials designed as storage materials for $\mathrm{H}_{2}, \mathrm{CO}_{2}$, and $\mathrm{CH}_{4}$, MOFs adsorbing benzene, alcohols, volatile organic compounds (e.g. toluene and other substituted benzenes), polyaromatic hydrocarbons, ammonia, and organophosphates are known. Fig. 1 shows a schematic diagram of possible cavities and sensing concept in MOF HKUST-1 [3].

Although this attractive potential is often well-known, little focus has been given to sensor applications. One of bottlenecks is that growing thin films of MOFs is a difficult process because they are neither soluble nor volatile. Typical solutions based deposition methods including spin coating, drop casting, liquid-phase epitaxial growth, CVD, ALD, sputtering, vapor phase epitaxial growth are not viable. Each MOF requires a specialized film growth procedure. Recently, there are several different reported methods for producing thin films of specific MOFs [4,5]
Our past work has demonstrated that the thin MOF HKUST-1 could be grown on a microcantilever surface and detect gases at room temperature, which was the first report of a MOF-integrated functional device as we know [9]. The step-by-step method using a series of immersion steps [4,5] was used to deposit thin HKUST-1 film on gold surface, yielding an average film thickness of $~ 100$ $\mathrm{nm} / 20$ cycles [9]. Recently, interdigital electrodes (IDE) were coated with several MOFs and tested in a range of $120-240^{\circ} \mathrm{C}$. The printable thick-film pastes which are mixed MOF powers with an organic vehicle were used to form $10 \mu \mathrm{m}$ thick films [10].

This paper focuses on stress induced sensing mechanism with two different MOFs, specifically HKUST-1 and $\mathrm{Cu}$ (NDC)DABCO. Our interest in MOFs was to explore the chemically induced stress produced by analyte adsorption on a microcantilever array sensor and to tailor the pore dimensions and chemical environment for improved selectivity. Microcantilever sensors were characterized using water vapor and methanol vapor in a custom designed gas test cell. The sensors exhibited substantially high sensitivity at room and elevated temperature. The long term goal of this work is to develop Stress induced microcantilever sensor system to provide selectivity and sensitivity for a broad range of analytes including explosives, nerve agents, and volatile organic compounds (VOCs).

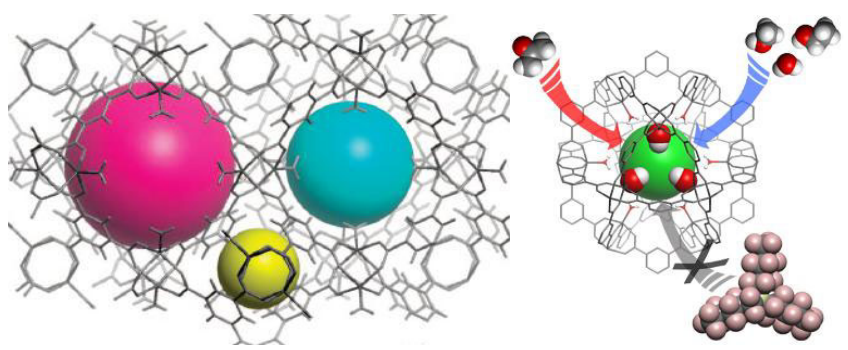

Figure 1. Schematic diagram of possible cavities and sensing concept in HKUST-1, a Cu-containing MOF, with $\sim 11.2 \AA$ diameter (left), $\sim 8.4 \AA$ diameter (right), and $\sim 5.4 \AA$ diameter (center). The pore size can be tailored for the adsorption of specific analytes [3].

\section{DESIGN AND FABRICATION Monte Carlo Modeling}

The more than 1000 new MOFs are reported annually. To screen MOFs for the potential use in chemical detection schemes, a comparison of analyte uptakes at trace concentrations is needed. To accomplish this we performed grand canonical Monte Carlo simulations, using the universal force field to simulate MOF-analyte interactions. The predicted volumetric uptake of each MOF at $10^{-6}$ $\mathrm{kPa}$ is shown in Figure 2. At this low pressure, short-range interactions between analyte and MOF should dominate over pore size or pore volume considerations. Several features characteristic of individual MOFs are noteworthy. First, CrMIL-53lp is the only MOF we considered that shows a significant uptake of xylenes, even at this low pressure. The small pores in CrMIL-53lp are lined 
with phenyl groups, creating an ideal environment for the adsorption of small aromatic molecules. Second, IRMOF-2, which contains the $\mathrm{Br}$ functional group, shows relatively high analyte uptake across the entire pressure range, with an exceptional uptake of GA compared to the other MOFs. Finally, the amine functional group in IRMOF-3 results in high uptake of heavier analytes, particularly the explosives class (RDX and TNT).

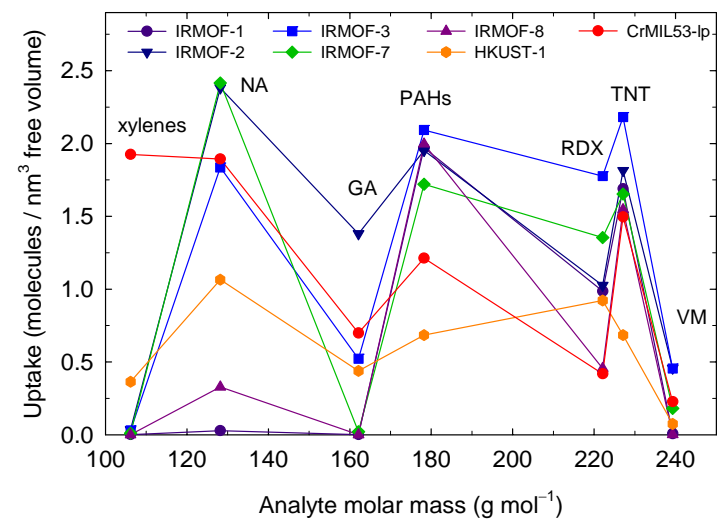

Figure 2. Uptake as a function of molar mass at a loading pressure of $10^{-6} \mathrm{kPa}$. Labels indicate each analyte type.

\section{MOF Coatings}

Figure 2 is schematically illustrated MOF coating setup. Thin films of the MOF HKUST- 1 and $\mathrm{Cu}$ (NDC)DABCO were grown on $300 \AA \mathrm{Cr} / 2000 \AA$ Au layers deposited microcantilever surfaces using thiol-based self-assembled monolayers (SAMs). SAMs layer was functionalized for $\sim 20$ hrs. For HKUST-1, there are a couple of key advantages. First, it is one of the known permanently porous MOFs with extensive studies into its properties and adsorption characteristics. Therefore, the behavior of HKUST-1 coated microcantilever can be expected. Second, there are well established methods for growing thin HKUST-1 films [4,5]. With respect to $\mathrm{Cu}$ (NDC)Dabco, there are known methods for growing thin film formed. It is reported to have enhanced flexibility over HKUST-1 to grow much closer to actual epitaxial growth, but there is not much literature on its properties. The layer-by-layer method was used for MOF film growth using a sequential repeating immersion paradigm [4,5]. Microcantilevers are immersed in a heated solution of $1 \mathrm{mM}$ $\mathrm{Cu}(\mathrm{OAc})_{2}$ in for HKUST-1 followed by immersion in a dilute solution of the MOF linker, $0.1 \mathrm{mM}$ benzene tricarboxylic acid (BTC) in ethanol. Ethanol was used as a washer followed each process. The cycle is repeated over multiple iterations to generate a uniform polycrystalline film. The process is automated through the use of peristaltic pumps programmed to alternately pump the desired solutions in and out of a reactor flow cell. The flow rate was $9 \mu \mathrm{L} / \mathrm{s}$ with $90 \mathrm{sec} /$ fill and $95 \mathrm{sec} /$ drain. The flow cell includes a heating jacket to allow the growth temperature $\left(50^{\circ} \mathrm{C}\right)$ to be controlled. The growth period for a 40 cycle was less than 26 hrs. The same method used for Cu(NDC)DABCO with $0.2 \mathrm{mM}$ (naphthalenedicarboxylate (NDC) and diazabicyclo[2.2.2]octane (DABCO). X-ray diffraction (XRD) and micro-surface-enhanced Raman spectroscopy ( $\mu$ SERS) were used to verify the presence of the MOF film on the substrate surface.

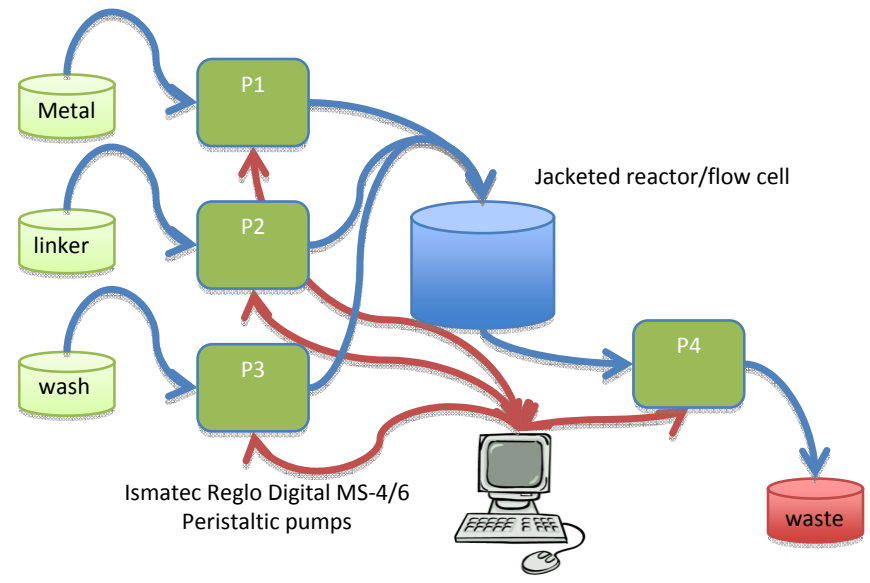

Figure 3. Schematic diagram of MOF coating setup.

\section{Fabrication}

N-type piezoresistive microcantilever arrays with $230 \mu \mathrm{m}$ in length and $100 \mu \mathrm{m}$ in width were successfully fabricated using batch microfabrication processes in Figure 4. While the advantages and fabrication process of this device (without MOFs) was described in [11], this paper includes an improved fabrication process and optimized sensor dimensions for gas detection with MOFs. Piezoresistive microcantilever arrays were initially formed from a silicon-on-insulator (SOI) wafer with (100) single crystal silicon (SCS) layer for the piezoresistive resistors. The SOI wafers were doped by ion implantation with phosphorus to create n-doped piezoresistors and then activated by annealing process. The silicon piezoresistors were defined by reactive ion etch (RIE) in Fig. 4a. A $\mathrm{Ti} / \mathrm{Au}$ was deposited as metal contacts to the resistors (Fig. 4b), and a thin layer of silicon nitride was carefully chosen to have a compressive stress and was deposited on top of these contacts as an insulating layer for the electrical components of the device and the piezoresistors (Fig. 4c). An aluminum layer was sputter-deposited and patterned to create a mask for the definition of the cantilevers and the chip. Then a low-stress silicon nitride was deposited on top of the aluminum mask to effectively strengthen the microcantilever area upon release (Fig. 4d). After the backside oxide as a mask was patterned, deep RIE through the wafer was used as a first release step (Fig. 4e). Finally, microcantilever arrays were released followed by RIE etching for the silicon nitride layer and the aluminum layer (Fig. 4f).

\section{Gas Testing}

The response of the sensor for $\mathrm{H}_{2} \mathrm{O}$, and $\mathrm{CH}_{3} \mathrm{OH}$ was measured in a custom designed gas test cell in Figure 5. Dry nitrogen was used as a carrier gas. Water and methanol were regulated using a hydrator with a mass flow controller 2 (MFC 2). The total gas volume was $100 \mathrm{sccm}$ with MFC 1 and 2 to keep the same flow rate. The stress response was obtained by the subtraction of the uncoated reference microcantilever response from the MOF-coated cantilever response using an AC Wheatstone bridge in order to remove the effect of temperature. The signal frequency was $10 \mathrm{kHz}$ and the time constant on the SR830 lock-in amplifier (SRS Instruments, Sunnyvale, CA) was 300 ms. A PC computer with LabVIEW for data acquisition was connected to a lock-in-amplifier and digital voltmeter for measurements. The microcantilever array chip was mounted in a designed stereolithography package for gas tests. 


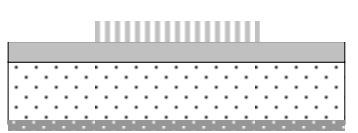

(a)

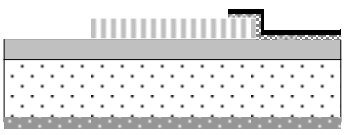

(b)

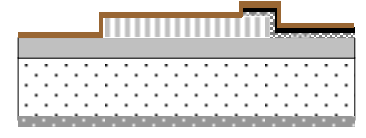

(c)

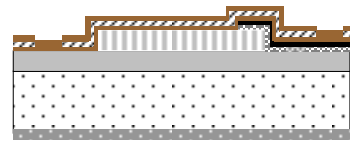

(d)

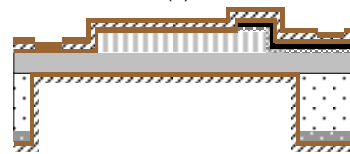

(e)

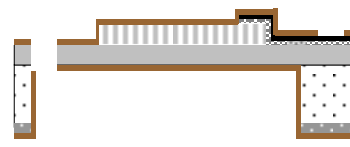

(f)

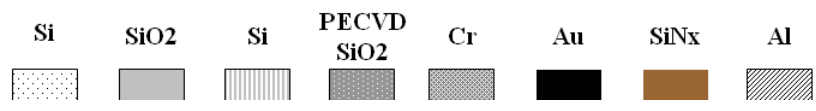

Figure 4. Batch piezoresistive microcantilever array sensor fabrication sequence. (a) Patterning piezoresistors on SOI wafer; (b) patterning conductive connections; (c) deposition of silicon nitride; (d) deposition of Al and silicon nitride as a mask; (e) backside etch through wafer by RIE followed by deposition of bottom silicon nitride and $\mathrm{Al}$; $(f)$ release of the piezoresistive microcantilever arrays by RIE etch.

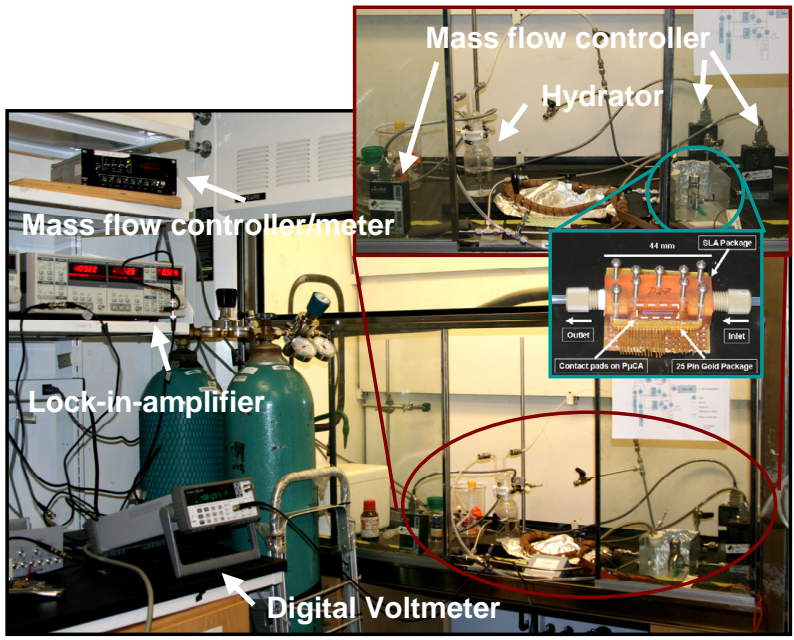

Figure 5. Schematic diagram of gas test setup.

\section{RESULTS AND DISCUSSION}

Microcantilevers with $230 \mu \mathrm{m}$ in length and $100 \mu \mathrm{m}$ in width were successfully fabricated in arrays of ten per chip, with 38 devices per a wafer using batch microfabrication technologies. The width and length of resistors are $20 \mu \mathrm{m}$ and $195 \mu \mathrm{m}$ in Fig. 6a and 6b. Thin MOF layer (Fig. 6c and 6d) is clearly visible on a microcantilever compared with uncoated cantilever (Fig. 6b). XRD and $\mu$ SERS were used to verify that MOFs are present on the surface (Fig. 7). Even though XRD can verify MOF film ( $>1 \mu \mathrm{m}$ thick), the microcantilever is too small to be characterized. We found that micro-surface-enhanced Raman spectroscopy ( $\mu S E R S$ ) is a viable characterization method for MOF films on microcantilevers with small spatial resolution $\left(<\sim 2.0 \mu \mathrm{m}^{2}\right)$ [9]. Figure $7 \mathrm{~b}$ shows the same SERS spectrums of HKUST-1 on a microcantilever (top), on a

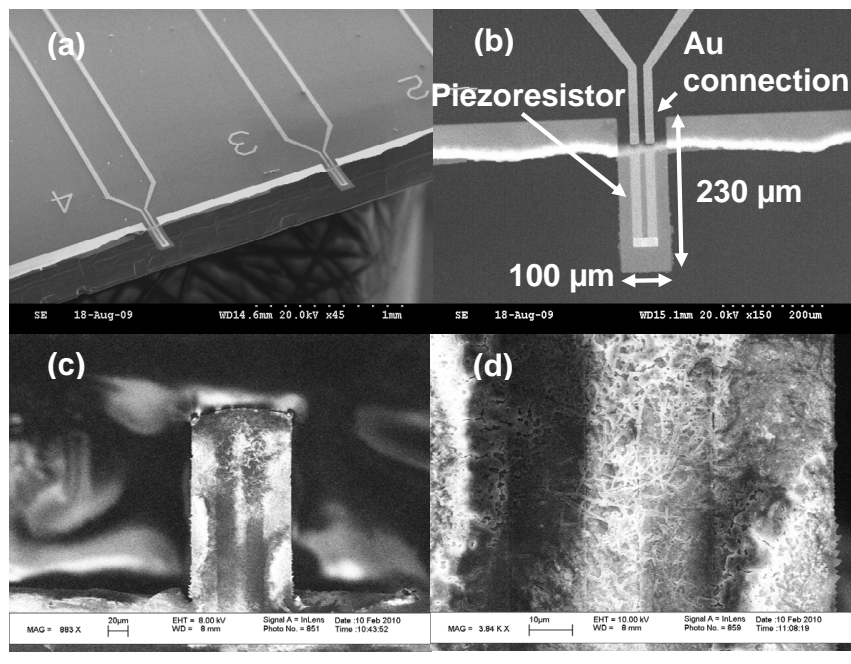

Figure 6. SEM of piezoresistive microcantilever. (a) each microcantilever array consisted of ten cantilevers; (b) reference microcantilever without HKUST-1 MOF coating; (c) and (d) HKUST-1 coated microcantilever sensor.

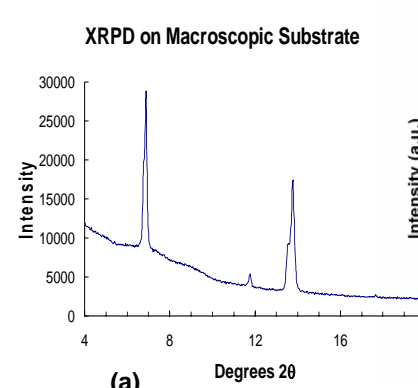

(a)

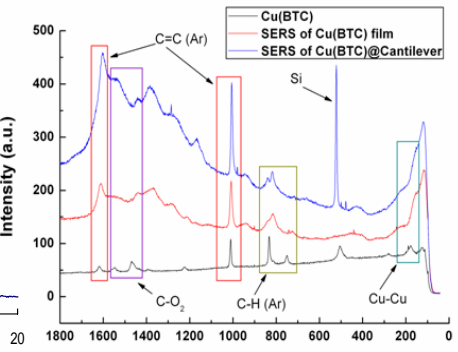

(b)
Figure 7. (a) Powder X-ray pattern of an oriented thick HKUST-1 film on a silicon substrate and (b) micro-surface-enhanced Raman spectroscopy ( $\mu$ SERS) pattern of an HKUST-1 film on a microcantilever [9].

macroscopic substrate (middle) and unenhanced Raman (without Ag layer) of a thick HKUST-1 layer as a reference (bottom).

MOF-coated microcantilevers exhibit substantial resistance changes and fast response times, with completely reversible response for adsorption and desorption of water and methanol, as seen in Fig. 8 and 9. HKUST-1 displays a higher resistance change for a given analyte concentration than $\mathrm{Cu}(\mathrm{NDC}) \mathrm{DABCO}$. Both the response and recovery times can be increased substantially by heating the sensor. For example, the response of the

$\mathrm{Cu}(\mathrm{NDC}) \mathrm{DABCO}$-coated sensor was 5 times faster at $35^{\circ} \mathrm{C}$ than at $20^{\circ} \mathrm{C}$ when a direct voltage of $1 \mathrm{~V}$ was applied to the heat cantilever (Fig. 9a). The measured response depends on concentration and conforms to Langmuir type saturation, as seen in Fig. 9b and 9c. The sensors exhibited a high sensitivity; for HKUST-1 coatings, a resistance change of $25.1 \mathrm{ohms} /$ Mole \% (at $1 \mathrm{~atm}$ ) was observed for $\mathrm{H}_{2} \mathrm{O}$ adsorption at room temperature. These results show that stress-induced piezoresistive microcantilever array sensors with nanoporous MOFs can provide a highly sensitive and reversible sensing mechanism for multiple analytes. This research is now being extended to test various gases such as $\mathrm{CO}, \mathrm{CO}_{2}, \mathrm{NO}, \mathrm{C}_{2} \mathrm{H}_{4}$, and $\mathrm{C}_{2} \mathrm{H}_{6}$ with various MOF compounds. 


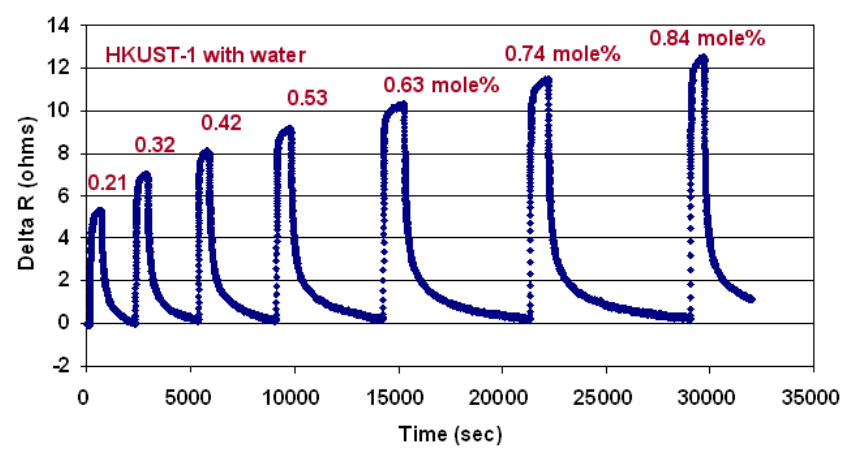

Figure 8. Response curves of HKUST-1 coated microcantilever sensors with water vapor at room temperature.
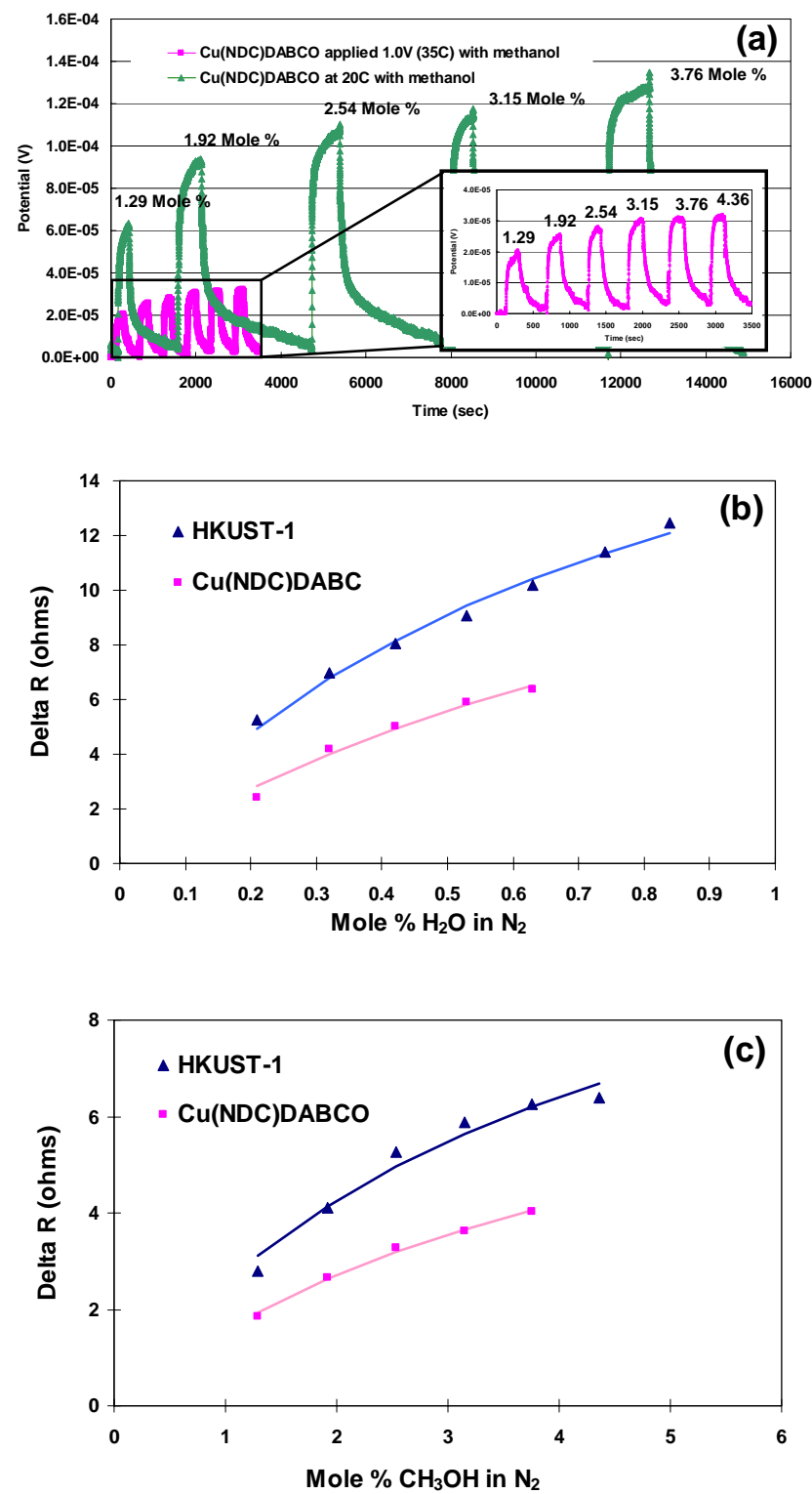

Figure 9. Characterization curves of microcantilever sensors. (a) Response time with $\mathrm{Cu}(\mathrm{NDC}) \mathrm{DABCO}$ at room and $35^{\circ} \mathrm{C}$ temperature; Langmuir curves for (b) water vapor and (c) methanol vapor at room temperature.

\section{CONCLUSIONS}

Stress induced piezoresistive microcantilever array sensors with nanoporous MOFs, HKUST-1 and Cu(NDC)DABCO, have been successfully demonstrated to provide a highly sensitive and reversible sensing mechanism. Thin films of MOFs were successfully grown with SAMs layer on microcantilever surface using the layer-by-layer method, which repeated a sequential immersion paradigm. MOF-coated microcantilevers exhibit substantial resistance changes and fast response times, with completely reversible response for adsorption and desorption of water and methanol. The response and recovery times on elevated temperature can be faster substantially than on room temperature. This successful development of MOFs coated microcantilever array sensor will enable a highly sensitive and reversible sensing mechanism for multiple analytes.

\section{ACKNOWLEDGEMENTS}

This work was supported by the Sandia Laboratory Directed Research and Development (LDRD) program. The authors would like to gratefully acknowledge Dr. Xiaohui Lin for his valuable technical assistance in device characterization.

\section{REFERENCES}

[1] S. S. -Y. Chui, S. M.-F. Lo, J. P. H. Charmant, A. G. Orpen, I. D. Williams, "A Chemically Functionalizable Nanoporous Material $\left[\mathrm{Cu}_{3}(\mathrm{TMA})_{2}\left(\mathrm{H}_{2} \mathrm{O}\right)_{3}\right]_{\mathrm{n}}$ ”, Science, 283, 1148 (1999).

[2] K. Uemura, Y. Komagawa, Y. Yamasaki, H. Kita, "Characterization of organic solvents adsorption desorption on hydrophobic porous coordination polymers and their micro-crystals aggregation on mullite support”, Desalination, 234, 1 (2008).

[3] K. W. Chapman, G. J. Halder, P. J. Chupas, “Guest-Dependent High Pressure Phenomena in a Nanoporous Metal-Organic Framework Material”, J. Am. Chem. Soc., 130, 10524 (2008).

[4] O. Shekhah, H. Wang, S. Kowarik, F. Schreiber, M. Paulus, M. Tolan, C. Sternemann, F. Evers, D. Zacher, R. A. Fischer, C. Wöll "Step-by-Step Route for the Synthesis of Metal-Organic Frameworks”, J. Amer. Chem. Soc., 129, 15118 (2007).

[5] D. Zacher, O. Shekhah, C. Woll et al., "Thin films of metal-organic frameworks”, Chemical Society Reviews, 38, 1418 (2009).

[6] E. Biemmi, C. Scherb, and T. Bein, "Oriented growth of the metal organic framework Cu3(BTC)2(H2O)3 xH2O tunable with functionalized self-assembled monolayers”, J. Am. Chem. Soc., 129, 8054 (2007).

[7] Q. M. Wang, D. M. Shen, M. Bulow et al., "Metallo-organic molecular sieve for gas separation and purification", Microporous Mesoporous Mater., 55, 217 (2002).

[8] S. Bordiga, L. Regli, F. Bonino et al., "Adsorption properties of HKUST-1 toward hydrogen and other small molecules monitored by IR”, Phys. Chem. Chem. Phys., 9, 2676 (2007).

[9] M. D. Allendorf, R. J. T. Houk, L. Andruszkiewicz, A. A. Talin, J. Pikarsky, A. Choudhury, K. A. Gall, P. J. Hesketh, "Stress-Induced Chemical Detection Using Flexible Metal-Organic Frameworks”, J. Am. Chem. Soc., 130, 14404 (2008).

[10]S. Achmann, G. Hagen, J. Kita, I. M. Malkowsky, C. Kiener, R. Moos, "Metal-Organic Frameworks for Sensing Applications in the Gas Phase”, Sensors, 9, 1574 (2009)

[11] A. Choudhury, P. J. Hesketh, Z. Hu, T. Thundat, “A piezoresistive microcantilevers array for surface stress measurement: Curvature model and fabrication”, $J$. Micromech. Microeng., 17, 2065 (2007). 